\title{
Fatigue and crack propagation investigations on composite dowels using an inclined single push-out test
}

\author{
K. Wolters ${ }^{\text {a* }}$, M. Feldmann ${ }^{\mathrm{a}}$ \\ ${ }^{a}$ Institute of Steel Construction, RWTH Aachen University, Germany \\ *corresponding author, e-mail address: k.wolters@stb.rwth-aachen.de
}

\begin{abstract}
The current fatigue design of composite dowels is based on the structure stress concept for the steel part and upper load limitations to avoid concrete fatigue and a degradation of the composite joint. Therefore the aim of the existing concept is to avoid any fatigue of the composite structure.
\end{abstract}

A fatigue concept considering residual load bearing capacity of torn steel connectors and the transfer of forces to less stressed composite dowels in the beam has great economic potential and leads to a better safety assessment. Therefore, further investigations of fatigue behaviour and crack propagation of composite dowels are necessary.

In a first step a single composite dowel is investigated in small-part tests. By the use of finite element models a new inclined single push-out test stand has been developed in order to reproduce the force and stress distributions within a composite beam as precisely as possible with the small-part tests. This ensures the comparability of the crack initiation location and crack propagation.

In the test series the influences of different stress ranges and cycle numbers on crack propagation of the steel dowel are investigated. Furthermore the residual load-bearing capacity is determined and compared to static load-bearing behaviour of uncracked steel parts of composite dowels. This paper concentrates on the numerical evaluation of crack propagation and residual capacity.

Keywords: Composite construction; composite dowel strip; fatigue; crack propagation; single push-out tests; FE analysis.

\section{Introduction}

In recent years, the use of composite dowels in bridge constructions became more and more popular. So far, 36 bridges [1] with composite dowels have been built in Europe. Since composite dowels are not standardized in Eurocode 4, they can be designed according to the German technical approval [2].

Especially for bridge constructions, the fatigue behaviour of the shear connectors is of great importance. The current concept for cyclic design is to avoid any fatigue of steel and concrete [3]. A concept which considers fatigue damage of concrete [4] and the residual loadbearing capacity and stiffness of torn steel dowels has a great economic potential. Therefore a knowledge of crack development in the steel dowel as well as of the redistribution of forces in the individual dowels of a composite beam is necessary.

In a first step, a single composite dowel with clothoid shape is investigated in single push-out tests (SPOT). Experimental and numerical studies investigate the crack propagation and residual load-bearing capacity.

\section{Fatigue crack propagation}

\subsection{Stress intensity factor $K$}

Components under cyclic loading are usually designed in a way that stresses stay in the elastic range. With the exception of the crack tip, where local plastic deformations may occur, this also applies to cracked steel dowels. The assumption 
of a linear-elastic and isotropic material behaviour leads to linear-elastic fracture mechanics. Based on the work of Westergaard, Irwin [5] introduced the stress intensity factor $K$. Therefore the stress field can be described by the function

$$
\sigma_{i j}=\frac{K}{\sqrt{2 \pi r}} f_{i j}(\theta)
$$

where $r$ and $\theta$ define the position of an infinitesimal element in the stress field (Fig. 1) and $f$ is a nondimensional trigonometric function. The stress intensity factor $K$ indicates the amount of increasing stresses near the crack tip $(r \rightarrow 0)$.

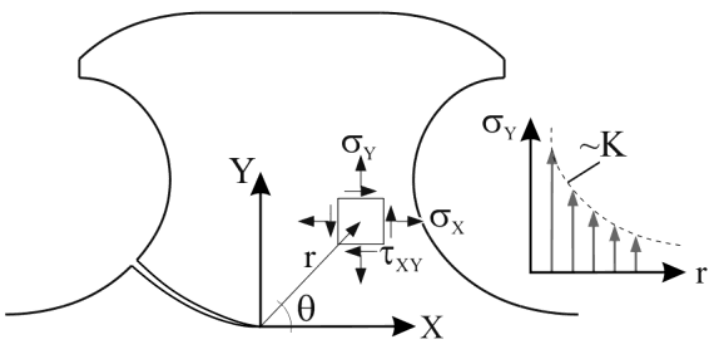

Fig. 1. Stress components at crack tip

Furthermore, the stress intensity factor can be divided into the three components tension perpendicular to the crack plane (Mode I, $K_{I}$ ), shear in longitudinal (Mode II, $K_{I I}$ ) and transversal direction (Mode III, $K_{I I I}$ ). For composite dowels the first two modes have to be considered (Fig. 2).

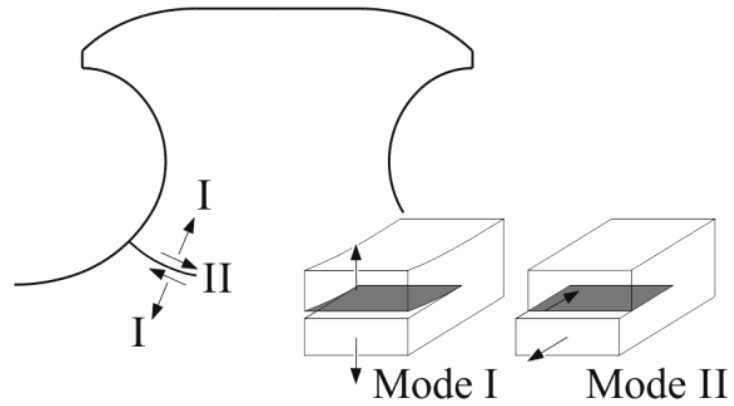

Fig. 2. Crack modes for composite dowels

The determination of $K$ or its components can be difficult since $K$ is dependent on crack length $a$, crack geometry (function $Y$ ) and geometry of the cracked component (function $M_{k}$ ):

$K=\sigma \sqrt{\pi a} Y M_{k}$

The analytical formulation of $Y$ and $M_{k}$ is limited to a few standard applications. Often these functions are determined experimentally or numerically [6]. For composite dowels there is no existing approximation formula, so that the stress intensity factors $K_{I}$ and $K_{I I}$ used in this paper are determined numerically, see chapter 4.2 .

\subsection{Crack development}

The stress intensity factors from fracture mechanics can also be used for fatigue crack propagation. Because of a linear dependence between $K$ and $\sigma$, a cyclic stress intensity $\Delta K$ can be easily found (Fig. 3).

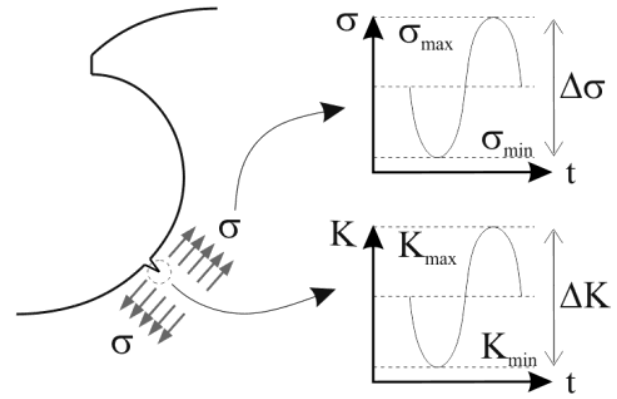

Fig. 3. Relation between stress and intensity factor

Using the cyclic stress intensity, the rate of crack propagation can be found according to the Paris Law [7]:

$d a / d N=C \Delta K^{m}$

$C$ and $m$ are material parameter. In a diagram with double-logarithmic scaling, the Paris Law describes a straight line (Fig. 4, right). It is valid for intermediate cyclic intensity factors leading to a stable crack development and is limited by no crack propagation in case of small intensity $\left(\Delta K<\Delta K_{t h}\right)$ and unstable crack propagation in case of high loads $\left(\Delta K>\Delta K_{c}\right)$. Formulas for these cases can be found in [8].
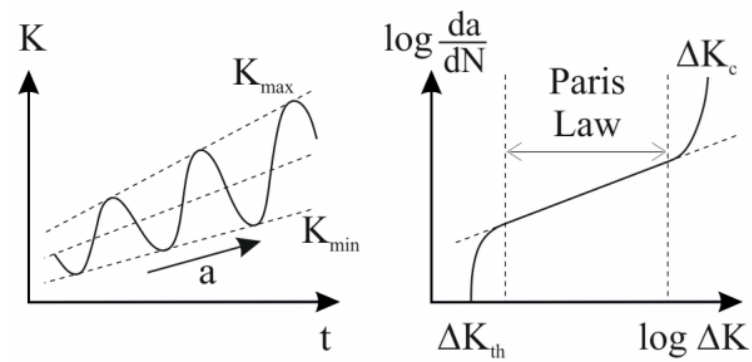

Fig. 4. Left: qualitative progression of $\Delta K$ with increasing crack length $a$; right: Paris straight in double-logarithmic scale 
The crack propagation direction $\hat{\theta}$ can be obtained using the condition $\tau_{r \theta}=0$.

$\theta=\cos ^{-1}\left(\frac{3 K_{I I}^{2}+\sqrt{K_{I}^{4}+8 K_{I}^{2} K_{I I}^{2}}}{K_{I}^{2}+9 K_{I I}^{2}}\right)$

\section{Test set-up}

\subsection{Transferability of small-part tests and beams}

A systematic investigation on large-scaled composite beams is not feasible for economic reasons. Therefore, a new test set-up for smallpart tests with a single composite dowel was developed to simulate a composite beam. The main problem finding an appropriate test set-up was to ensure stability against internal moments in an asymmetric specimen on the one hand and to reach a stress distribution in the steel dowel similar to those of a beam on the other hand. With the help of numerical simulations an angled single push-out test (SPOT), where load introduction, resulting force transmission and reaction force have the same line of action, was developed in [9]. Fig. 5 shows the test set-up and compares the maximum principal stress distributions of steel dowels in composite beams and SPOT. It can be shown that the areas of maximum tensile stresses coincide so that the theoretical location of crack initialisation matches.

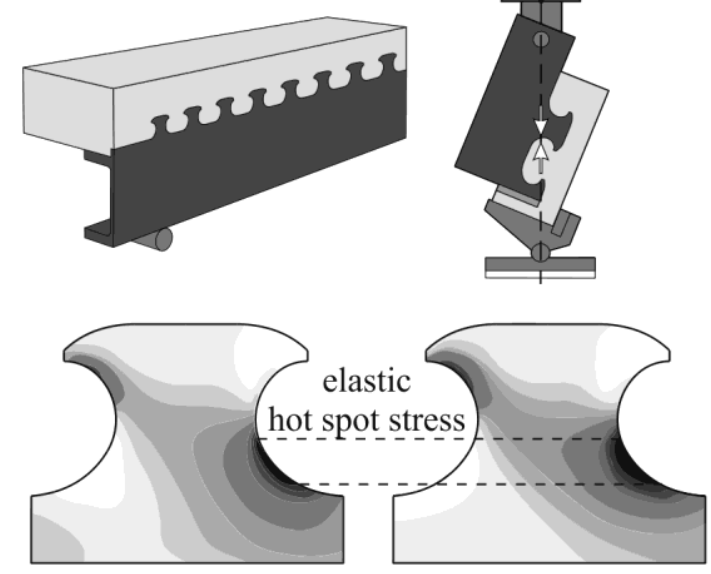

Fig. 5. Comparison of elastic maximum principal stress distribution in composite beams and SPOT

\subsection{Test programme and present results}

As part of a German national research project, 15 test specimens were produced for the angled SPOT, to investigate fatigue, crack development and residual load-bearing capacity. The steel dowel with clothoid shape and a dowel spacing of $e_{x}=200 \mathrm{~mm}$ was made from flat steel, grade S355 by gas cutting. To avoid concrete failure, a concrete strength class C50/60 with a high degree of reinforcement was used. Strain gauges were applied to the steel dowel in the hot spot zone to analyse the stresses and to calibrate finite element models (Fig. 6). The second, halved steel dowel forms the concrete dowel and does not influence the load-bearing capacity due to a polystyrene gap filler.

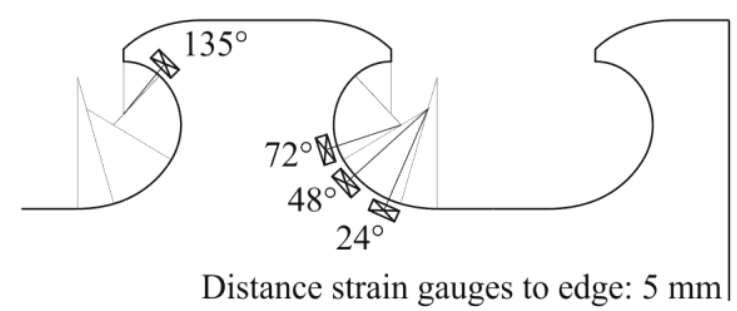

Fig. 6. Arrangement of strain gauges

The first test specimen was tested as a reference in a static path-controlled test, till the halved steel dowel overclosed the gap. Until then a slip between steel and concrete surface of about $22 \mathrm{~mm}$ at a maximum load of $717 \mathrm{kN}$ could be measured. At that time, no drop in the load and no failure could be observed. The resulting loadslip behaviour of the composite dowel can be found within the calibration of the FE model in chapter 4.1. The maximum strains and therefore stresses could be measured on the middle strain gauge, which corresponds to the aspired hot spot location.

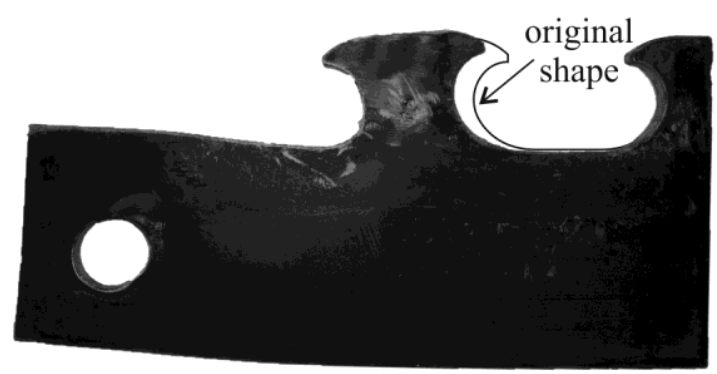

Fig. 7. Deformed steel dowel after static reference test

Using the results of the static reference test, load limits for further tests were set. The 14 cyclic tests are divided into two work packages. First, the influence of different web thicknesses on crack propagation and residual load-bearing capacity is to be investigated. The second work 
package examines different load limits and ranges with regard to crack development.

In the first two tests carried out so far no fatigue crack could be generated, because the preliminary design using the Wöhler concept was too much on the safe side. The focus in this paper is therefore on numerical investigations.

\section{Numerical investigation of crack development}

\subsection{Static reference test}

All numerical simulations are performed with the commercial finite element software ABAQUS. The static reference test was used to validate the FE model which is the starting point for simulations of crack propagation and residual load-bearing capacity. The load-slip behaviour of both is shown in Fig. 8 .

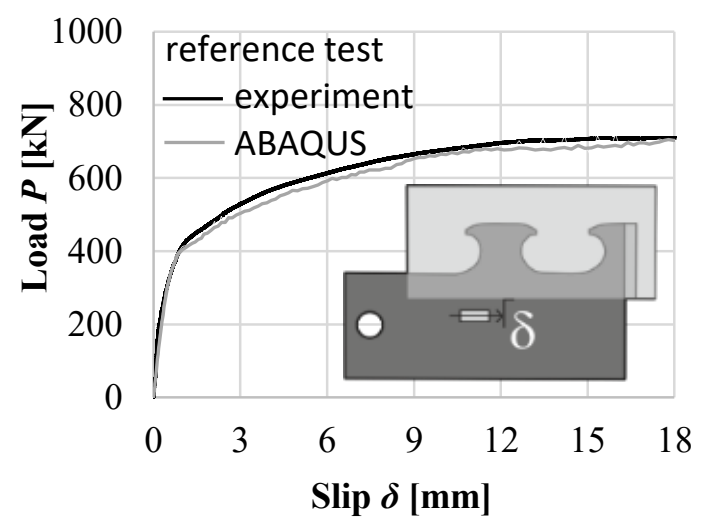

Fig. 8. Load-slip behaviour from static reference test and FE model

For the reference simulation as well as the determination of residual capacity the ABAQUS/Explicit solver is used to overcome convergence problems due to large plastic deformations. The model consists of the test specimen, the load introduction and the support. Volume elements (C3D8R) are used for each part. The reinforcement is embedded in the concrete part of the model. This function couples the deformation of nodes so that no slip between concrete and reinforcement is considered. Contact formulation with a hard contact in normal direction and a friction coefficient of 0.3 in tangential direction describes the interactions between the other parts. Since concrete damage is not of interest in detail, ideal elastic-plastic material models are used for concrete and reinforcement, while the material model of the steel is based on the actual stress-strain curve. As in the experiments, the loading is path-controlled and applied over boundary conditions. The plastic deformation of the steel dowel shown in Fig. 7 can be simulated well, Fig. 9 .

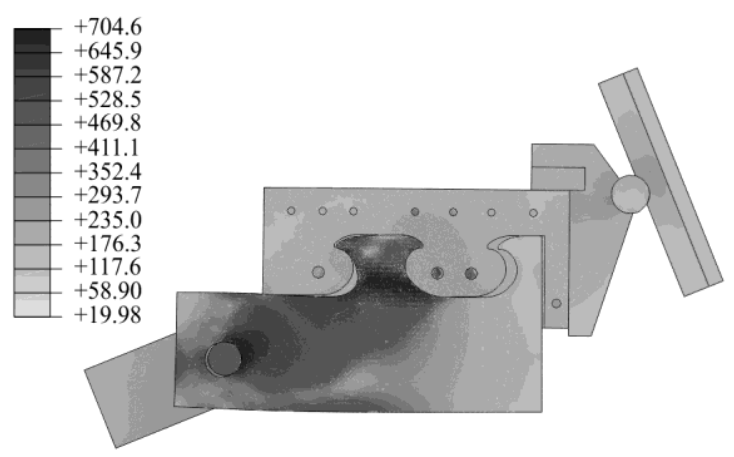

Fig. 9. Numerical simulation of reference test (Mises stress plotted; here at $\delta=15 \mathrm{~mm}$ )

\subsection{Crack propagation simulation}

To simulate cracks in the steel dowel, some adjustments have to be made in the numerical model. The calculation of stress intensity factors efforts a linear material behaviour. Also geometric nonlinearities are neglected. Unlike before, the ABAQUS/Standard solver is used.

ABAQUS is only able to calculate stress intensity factors in the static case and does not include automatic crack propagation. This problem is solved by using a Python based script which is explained later. For a calculation of stress intensity factors the followings steps are necessary [10]:

- The crack geometry is built in as a usual Partition in the steel dowel, like the partitions used for mesh control. Therefore, the crack is sketched within the function Partition face: Sketch in the Part module. Afterwards the sketch has to be extruded through the thickness of the steel dowel (Partition Cell: Extrude/Sweep Edges).

- The crack tip leads to numerical singularities. A special mesh technique is used to reach reasonable results [6]. Therefore, a few circular partitions are sketched around the crack tip with the methods described before (Fig. 10).

- The elements in the inner cycle have to be assigned as wedge elements (Fig. 10). This can be done with the function Assign Mesh Controls in the Mesh module. 


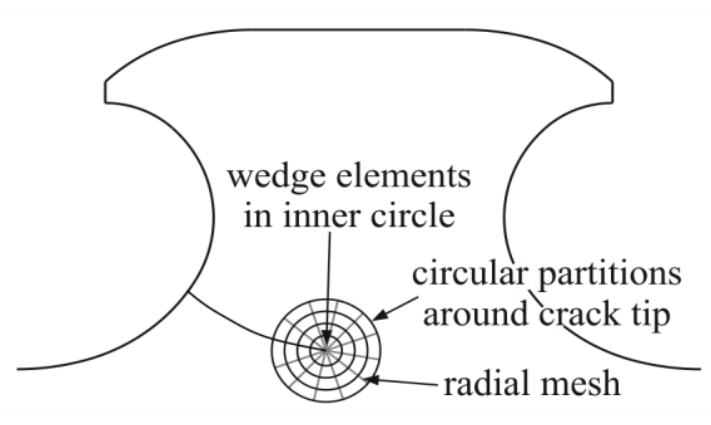

Fig. 10. Mesh technique for crack simulation

- Once these settings are made, the Part of the steel dowel has to be switched to independent. Now the mesh refers to the Instance steel dowel.

- The function Assign Seam to create a doubled surface along the existing crack can be found in the Interaction module (Special $\rightarrow$ Crack)

- The crack tip can be defined in the Assembly module using Engineering Features $\rightarrow$ Cracks. For the Contour integral method, a crack front and a crack extension direction have to be defined. Furthermore, to handle the singularity the midside node parameter is set to 0.25 and the Degenerate Element Control is set to Collapsed element side, single node.

- Finally, the history output of the crack can be gained. The Number of contours corresponds to the number of circular partitions in addition to the crack tip, which is the first contour where $K$ factors are calculated. The Type is set to Stress intensity factors with Maximum tangential stress as the Crack initiation criterion.

With these settings, ABAQUS considers the crack and calculates the three components of the stress intensity factor as well as the crack propagation direction. It is to be mentioned that the stress intensity factors are calculated and differ for each contour and each mesh plane over the thickness of the steel dowel. Especially the results for the outer mesh plane, for the crack tip (contour 1) and for the last contour are slightly different from the other results. Therefore, several contours and mesh planes are recommended to judge results in a better way.

A script based on Python is used to simulate crack propagation. The most important steps are summarized in Fig. 11. The main idea is to take an initial model without crack as a starting point. An elastic calculation determines the location of maximum tensile stresses in the hot spot for crack initiation. The crack geometry is defined by a text file, where coordinates for sketching the crack in the Part module are stored. Then the crack propagates over several incremental elastic calculations. After each simulation, stress intensity factors are stored for further calculations and the crack propagation angle is used to update the crack geometry.

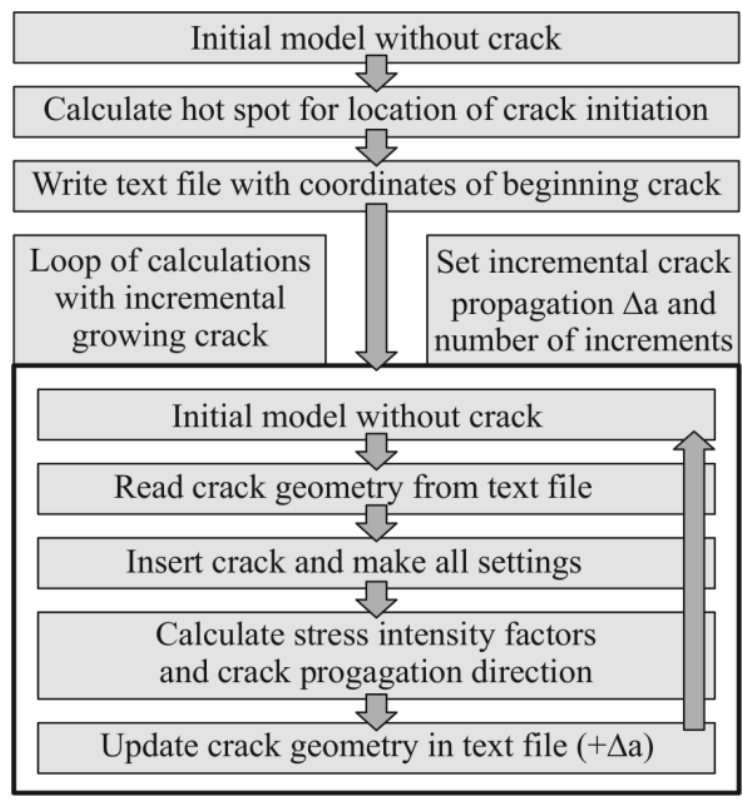

Fig. 11. Schematic process of crack propagation simulation

This method was used with the SPOT model. Overall 30 elastic simulations with a crack propagation increment of $3 \mathrm{~mm}$ led to the crack geometry shown in Fig. 12. The crack initially propagates nearly perpendicular to the steel dowel surface in the rounding. As soon as it reaches the middle of the steel dowel, it bends slightly upwards. The development of stress intensity factor $K_{I}$ at upper load ( $P=280 \mathrm{kN}$, as planned for experiments) is also displayed in Fig. 12.

The stress intensity factor increases with growing crack up to about $60 \mathrm{~mm}$ crack length. This coincides with an increase in the rate or velocity of crack propagation. The component $K_{I I}$ is about one percent of $K_{I}$ and neglected in further calculations. Furthermore, equation (2) is modified to be dependent on $P$ :

$$
K=K_{I}=\frac{P}{t_{w} e_{x}} \sqrt{\pi a} Y(a)
$$




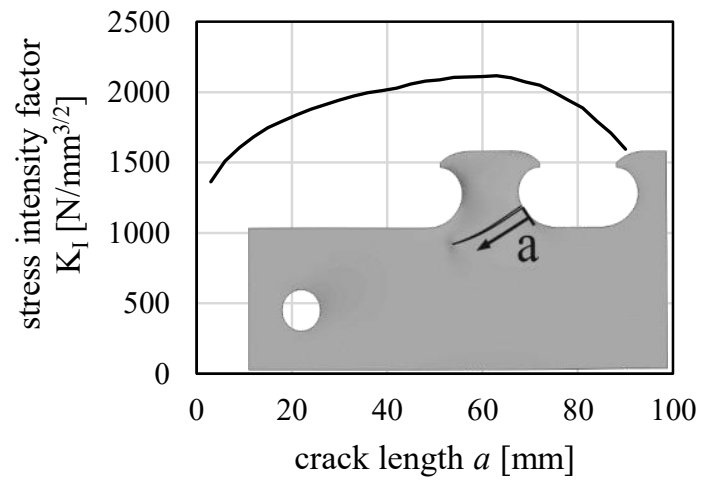

Fig. 12. Crack geometry after 30 increments and stress intensity factors $K_{I}$ for chosen maximum load $P=280 \mathrm{kN}$

Now the geometry factor $Y(a)$ can be easily calculated. A simple approximation is shown in Fig. 13.

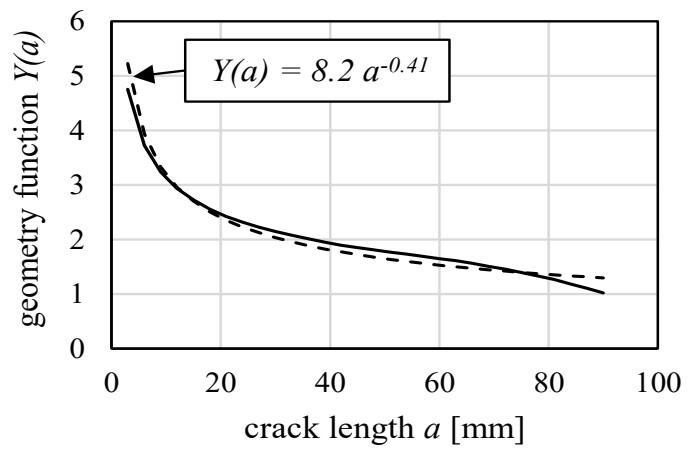

Fig. 13. Geometry function $Y(a)$ from numerical investigations and approximation
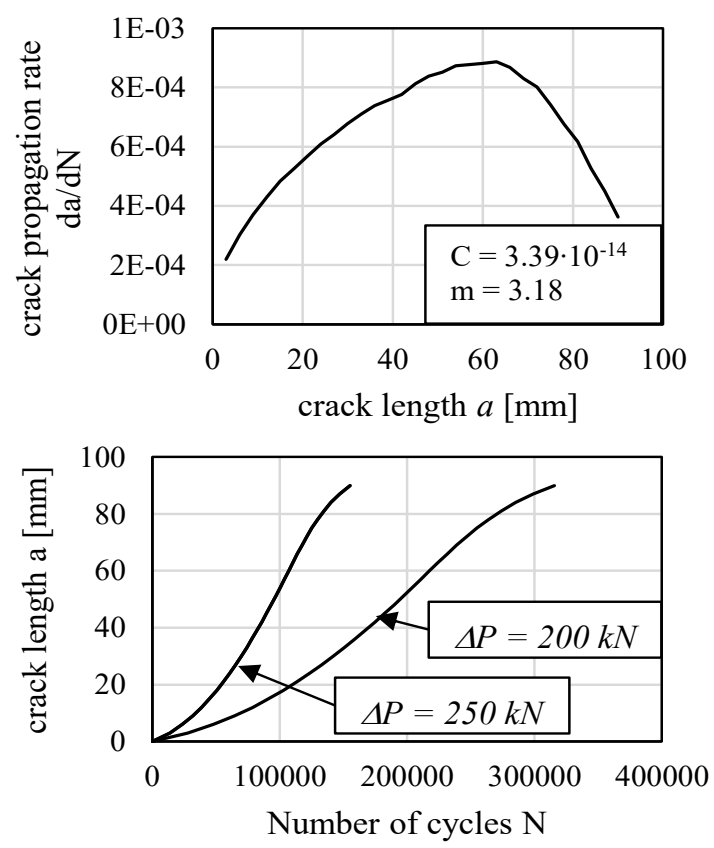

Fig. 14. Crack propagation rate for material parameters (S355) given in [11] (top); prediction of lifetime after crack initiation (bottom)
Finally, the rate of crack propagation can be calculated and lifetime can be predicted (Fig. 14). A sensitivity analysis shows a great dependence on the material parameters used in the calculations. In literature, values for the structural steel material parameter $m$ between 2.5 and 4 can be found [11]. Maintaining all other values, the lifetime prediction varies between 367 cycles $(m=4)$ and 23.6 million cycles $(m=2.5)$. So a lifetime prediction without knowing exact material parameters is impossible. They will be determined when the experimental test results are available.

\subsection{Residual load-bearing capacity}

The simulations on residual capacity are based on the calibrated FE model of the reference test described in chapter 4.1. The crack geometry is taken from the text file for crack propagation, see chapter 4.2. The results for certain crack lengths are plotted in Fig. 15. As an example, Fig. 16 shows the deformation in ABAQUS of the SPOT with a crack length of $60 \mathrm{~mm}$.

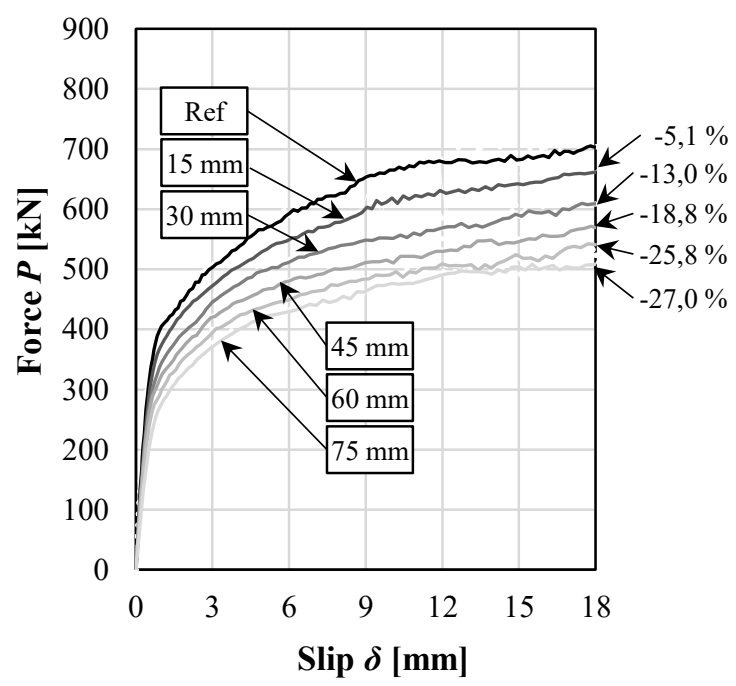

Fig. 15. Residual capacity of composite dowel with crack in steel dowel for different crack lengths $a$

As expected, the residual capacity decreases with increasing crack length. Like in the reference simulation, no drop in the load can be observed till the halved steel dowel overcloses the gap. The degradation of capacity can be taken from the diagram, the stiffness of the composite dowel decreases by 3.8 percent $(a=15 \mathrm{~mm})$ to 34.0 percent $(a=75 \mathrm{~mm})$. 


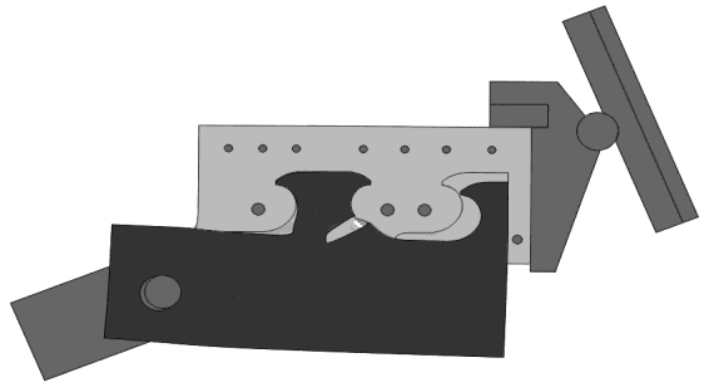

Fig. 16. Deformation of SPOT specimen for crack length $60 \mathrm{~mm}$

The results can only be taken as a first qualitative estimation of residual capacities and stiffness degradation, since so far no experimental results are available. An extension of the material model for steel to consider further shear damage in form of ductile crack propagation due to high loads seems reasonable. Also the effects of concrete damage due to the cyclic loading is not yet considered. A further reduction of residual capacities is expected. Having experimental results, the models will be calibrated.

\section{Conclusions and Outlook}

A new test set-up for single push-out tests was developed to simulate a single composite dowel of a composite beam. With this set-up a good agreement in the distributions of maximum tensile stresses between beam and small-part test can be achieved. The SPOT is used to investigate the fatigue, crack propagation and residual loadbearing capacity of steel dowels. This paper summarizes the theory of crack propagation and prediction of lifetime. Since experimental results are not yet available, the focus is on the numerical implementation and handling of cracks in ABAQUS.

It is shown that a crack propagation simulation in ABAQUS is possible by performing several incremental elastic calculations. The crack geometry seems reasonable but has to be evaluated when experimental results are available. Also the prediction of lifetime is difficult without knowing material parameters $C$ and $m$, because results are very sensitive for parameters given in literature. The investigations on residual capacities will be extended by material models for shear damage. Both will be calibrated to experimental results.
The findings on the small-part tests will be transferred to composite beams. The proposed concept for crack propagation simulation can be applied to composite beams too. Fig. 17 shows a crack propagation simulation on a composite beam in ABAQUS. Every dowel gets its own crack geometry as a text file. Even different rates of crack propagations can be considered by analyzing the stress intensity factors in each dowel. In further nonlinear simulations, a redistribution of forces in the steel dowels of a composite beam for different crack lengths can be determined. Three experimental tests on composite beams are part of the project.

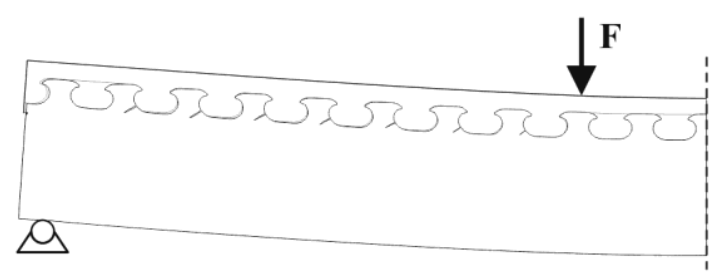

Fig. 17. Crack propagation simulation with beam model in ABAQUS

Aim of these investigations is a concept to consider fatigue and cracks in the design of composite dowels. An extension of the current concept which does not prevent fatigue damage has a great economic potential. Therefore, further investigations on residual capacities as well as on the redistribution of forces in a composite beam are necessary.

\section{References}

[1] Lorenc W: The model for a general composite section resulting from the introduction of composite dowels. Steel Construction 2017; 10:154-167.

[2] DIBt: Zulassung - Z-26.4-56 - Verbunddübelleiste 2013.

[3] Feldmann, M, Kopp M, Pak D: Composite dowels as shear connectors for composite beams - background to the German technical approval. Steel Construction 2016; 9:80-88.

[4] Claßen M, Gallwoszus J: Concrete fatigue in composite dowels. Structural Concrete 2016; 17:63-73.

[5] Irwin GR: Fracture. In: Flügge S. (Hg.): Handbuch der Physik. Elasticity and Plasticity. Berlin Heidelberg: Springer-Verlag. 1958;551590.

[6] Kuna M: Numerische Beanspruchungsanalsye von Rissen. Finite Elemente in der Bruchmechanik. s.l.: Vieweg+Teubner (GWV) 2008. 
[7] Paris PC, Gomez MP, Anderson WF: A Rational Analytic Theory of Fatigue. The Trend in Engineering 1961; 13:9-14.

[8] Haibach E: Betriebsfestigkeit. Verfahren und Daten zur Bauteilberechnung. Düsseldorf: VDIVerl. 1989.

[9] Wolters K, Kopp M, Feldmann M: Entwicklung eines neuen Single-Push-Out-Versuchsstands zur zyklischen Untersuchung der Verbunddübelleiste. Stahlbau 2017; 86:10981105.

[10] Dassault Systemes: Abaqus/CAE User's Guide, Chapter 31.2.

[11]Radaj D: Ermüdungsfestigkeit. Grundlagen für Leichtbau, Maschinen- und Stahlbau. Berlin: Springer 2003. 